\title{
Correção cirúrgica para reversão de operação de Senning em caso de falência ventricular direita
}

\author{
Adib D. JATENE*, Fábio B. JATENE*, Marcelo B. JATENE*, N. IKARI*, Deipara M. A. MACHADO*
}

\section{RBCCV 44205-132}

JATENE, A. D.; JATENE, F. B.; JATENE, M. B.; IKARI, N.; MACHADO, D. M. A. - Correção cirúrgica para reversāo de operação de Senning em caso de falência ventricular direita. Rev. Bras. Cir. Cardiovasc., 6(1): 49-53, 1991.

RESUMO: Os autores apresentam uma nova proposta para tratar disfunção do ventrículo direito no pós-operatório tardio da transposiçāo das grandes artérias associada a grande comunicação interventricular e que tenha sido tratada pela inversão em nivel atrial. Apresentam o caso de um paciente com 12 anos de idade, que havia sido submetido a operação de Senning associada a fechamento de comunicação interventricular e alívio de estenose pulmonar há 8 anos. A operação de Senning foi desfeita, restabelecendo-se a concordância atrioventricular, o remendo que fechava a comunicação interventricular foi ressecado, conectando-se a aorta com o ventrículo esquerdo. A artéria pulmonar foi seccionada transversalmente, próximo ao plano valvar. O coto proximal foi suturado e o distal, conectado ao ventrículo direito através de tubo valvulado de pericárdio bovino. A evoluçăo foi satisfatória, revertendo-se a situação do paciente do grupo funcional IV para grupo $\mathrm{I} / \mathrm{II}$.

DESCRITORES: Senning, operação de, reversāo; Senning, operação de, complicaçōes tardias; falência ventricular, cirurgia.

\section{INTRODUÇĀO}

A disfunção ventricular direita é complicação que pode ocorrer no pós-operatório tardio de pacientes com transposição das grandes artérias operados pelas técnicas de MUSTARD ${ }^{5}$ ou de SENNING ${ }^{8}$.

Esta situação representa um problema de difícil resolução, onde uma das opções terapêticas é o transplante cardíaco.

$\mathrm{MEE}^{4}$, conseguiu desfazer a operação em nível atrial, restabelecendo as concordâncias atrioventriculares e realizar a correção em nivel arterial.
Recentemente, tivemos a oportunidade de desfazer uma operação de Senning e realizar a correção pela técnica de Rastelli (RASTELLI et alii ${ }^{6}$ ).

\section{RELATO DO CASO}

D. J. M., do sexo masculino, procurou o Serviço em fevereiro de 1990, com 12 anos de idade e história pregressa de cianose e dispnéia progressiva desde o nascimento. Aos quatro anos de idade, com diagnóstico de transposição das grandes artérias, grande comunicação interventricular (CIV) e estenose pulmonar (EP), foi submetido a alívio da EP, fechamento da CIV, deixando a aorta no ventrículo direito, e inversão atrial pela

Trabalho realizado no Instituto do Coração do Hospital das Clínicas da Faculdade de Medicina da Universidade de São Paulo. São Paulo, SP, Brasil.

Apresentado ao 18: Congresso Nacional de Cirurgia Cardiaca. Rio de Janeiro, RJ, 5 e 6 de abril, 1991

* Do Instituto do Coraçăo do Hospital das Clínicas da Faculdade de Medicina da Universidade de Săo Paulo.

Endereço para separatas: Adib D. Jatene. Av. Dr. Enéas de Carvalho Aguiar, 44. Conselho Diretor. 03403 São Paulo, SP, Brasil. 
JATENE, A. D.; JATENE, F. B.; JATENE, M. B.; IKARI, N.; MACHADO, D. M. A. - Correção cirúrgica para reversão de operação de Senning em caso de falência ventricular direita. Rev. Bras. Cir. Cardiovasc., 6(1): 49-53, 1991.

técnica de Senning. Na descrição, está relatado que a aorta estava anterior e à esquerda do tronco pulmonar. Havia justaposição das aurículas à esquerda. Devido a esse fato, o átrio direito era pequeno. A canulaçāo foi feita nas duas veias cavas, usando-se hipotermia moderada. Após atriotomia direita, verificou-se pequena comunicação interatrial e grande comunicação interventricular, sendo difícil a diferenciaçāo entre transposiçāo e dupla via de saída de ventrículo direito. Após abertura do ventrículo direito, observou-se que a CIV era do tipo basal anterior. Havia EP do tipo anel fibroso, que foi ressecado através da CIV. Esta foi fechada com placa de Dacron. A seguir, foi realizada operaçāo de Senning com o auxilio de dois remendos de dura-máter, um pequeno para completar o septo atrial e outro grande para ampliar o átrio direito. Não foram relatadas quaisquer intercorrências no pós-operatório, permanecendo o paciente em ritmo sinusal. A evoluçāo foi satisfatória até ínicio de 1990, portanto oito anos, quando começou a apresentar dispnéia aos esforços, motivo da internação no Serviço. $\mathrm{Na}$ investigaçāo, constatou-se que, ao lado de disfunção do ventrículo direito e insuficiência tricúspide, tinha severa estenose infundibulo-valvar, com gradiente entre ventrículo esquerdo e tronco pulmonar de $142 \mathrm{mmHg}$. Foi, por isso, em abril de 1990, submetido a valvoplastia pulmonar com cateter balāo ${ }^{7}$. Apesar de ter havido queda do gradiente para $62 \mathrm{mmHg}$, não foi assinalada melhora clínica. Ao contrário, o paciente evoluiu com agravamento da dispnéia que, apesar da medicaçāo, chegou aos mínimos esforços. Em novembro de 1990 , encontrava-se em classe funcional IV, com regular estado geral, taquidispnéico, descorado + , com freqüência cardiaca de 120 batimentos por minuto e freqüência respiratória de 36 por minuto e pressāo arterial de 80 $\times 50 \mathrm{mmHg}$. Apresentava estase jugular de + a 45 graus, com frêmito sistólico + com borda esternal esquerda. O ictus era palpável no $5^{\circ}$ espaço intercostal esquerdo, para fora da linha hemiclavicular. As bulhas eram normofonéticas com B3 presente, ritmo de galope e manobra de Ribero Carvalho positiva +. Apresentava, ainda, sopro sistólico ++ e sopro diastólico ++ em borda esternal esquerda à altura do $2^{\circ}$ e 3 . espaços intercostais. O fígado era palpável a $2 \mathrm{~cm}$ do rebordo costal. O eletrocardiograma revelou ritmo sinusal, bloqueio de ramo direito e sobrecarga ventricular direita. O ecocardiograma mostrou insuficiência tricúspide importante, com estenose pulmonar infundíbulo-valvar e mau desempenho do ventrículo direito. $\mathrm{O}$ cateterismo cardíaco revelou as pressões descritas na Tabela 1. Após o cateterismo, apresentou edema agudo dos pulmōes de difícil reversão. O paciente foi mantido com drogas vaso-ativas (dobutamina $6 \mu \mathrm{g} / \mathrm{kg} /$ minuto, dopamina $3 \mu \mathrm{g} / \mathrm{kg} /$ minuto, furosemide $2 \mathrm{mg} / \mathrm{kg} /$ dia e captopril $0,89 \mathrm{mg} / \mathrm{kg} / \mathrm{dia}$ ). A operaçāo foi realizada em 12.12.90, por esternotomia mediana. A circulação extracorpórea foi feita por duas cânulas colocadas nas cavas através do átrio direito e uma cânula arterial colocada na artérial femoral comum direita. Foi, inicialmente, realizada a reversāo da operação de Senning com reconstruçāo do átrio esquerdo, utilizando-se o teto do túnel das cavas. A reconstrução do átrio direito exigiu o emprego de amplo remendo de pericárdio bovino preservado em glutaraldeído, com o qual se refez toda a sua parede lateral. O seio coronário foi deixado no átrio direito. A aorta estava anterior e à direita do tronco pulmonar, que era mais calibroso e onde se palpava frêmito sistólico. $O$ ventrículo direito foi aberto no mesmo local da cirurgia prévia, longitudinalmente, na sua via de saida. O retalho de Dacron que servia para o fechamento da CIV tinha cerca de $2 \mathrm{~cm}$ de diâmetro e estava completamente endotelizado. A CIV foi reaberta pela ressecção do Dacron (Figura $1 \mathrm{~A})$, ampliando-se um pouco na sua borda ântero-superior. Um novo remendo, agora de pericárdio bovino,foi suturado de tal forma que a aorta ficasse conectada ao ventrículo esquerdo (Figura $1 \mathrm{~B}$ ). $\mathrm{O}$ tronco pulmonar que havia sido seccionado transversalmente, para se avaliar a estenose pulmonar, quando se constatou rotura de uma das válvulas da valva, que era bivalvulada, e redução do anel com estenose subvalvar não tratável - foi totalmente seccionado. O coto proximal foi suturado (Figura 2) e o distal, ligado ao ventrículo direito por um tubo valvulado de pericárdio bovino de $20 \mathrm{~mm}$ de diâmetro (Figura 3). Não houve intercorrência digna de registro na saída de circulação extracorpórea. A pressāo do átrio esquerdo manteve-se em níveis normais durante todo o período de pós-operatório, que transcorreu sem anormalidades. As drogas vaso-ativas foram retiradas no 4: dia após a operaçāo e o paciente recebeu alta cirúrgica com 15 dias. Houve nítida redução da congestāo vascular pulmonar aos raios $\mathrm{X}$. O eletrocardiograma mantinha o bloqueio de ramo direito e apresentava raras extrassístoles. O ecocardiograma, no dia 8.1.91, mostrou tubo valvulado sem sinais de disfunção, insuficiência tricúspide discreta e desempenho ventricular esquerdo comprometido, porém melhor que no pósoperatório imediato. O cateterismo cardiaco realizado com duas semanas de pós-operatório revelou os dados constantes da Tabela 2.

TABELA 1

\begin{tabular}{lcrl}
\hline AD & - & 19 & 15 \\
VD & 57 & 7 & 10 \\
AP & 51 & 30 & 39 \\
CP & - & 21 & 18 \\
VE & 116 & 11 & 18 \\
Ao & 112 & 86 & 98 \\
\hline
\end{tabular}

TABELA 2

\begin{tabular}{lrrrrrr}
\hline & \multicolumn{2}{c}{ Repouso } & \multicolumn{5}{c}{ Pós ventriculografia D } \\
\hline AD & 9 & 10 & 8 & 9 & 8 & \\
VE & 97 & 0 & 5 & & & \\
VE alto & 73 & 0 & 6 & CP & 60 & 45 \\
AP & 60 & 30 & 41 & 81 & 35 & 55 \\
VD & 85 & 5 & 20 & 100 & 12 & 45 \\
Ao & 69 & 54 & 64 & 98 & 75 & 79 \\
\hline
\end{tabular}


JATENE, A. D.; JATENE, F. B.; JATENE, M. B.; IKARI, N.; MACHADO, D. M. A. - Correção cirúrgica para reversão de operação de Senning em caso de falência ventricular direita. Rev. Bras. Cir. Cardiovasc., 6(1): 49-53, 1991.
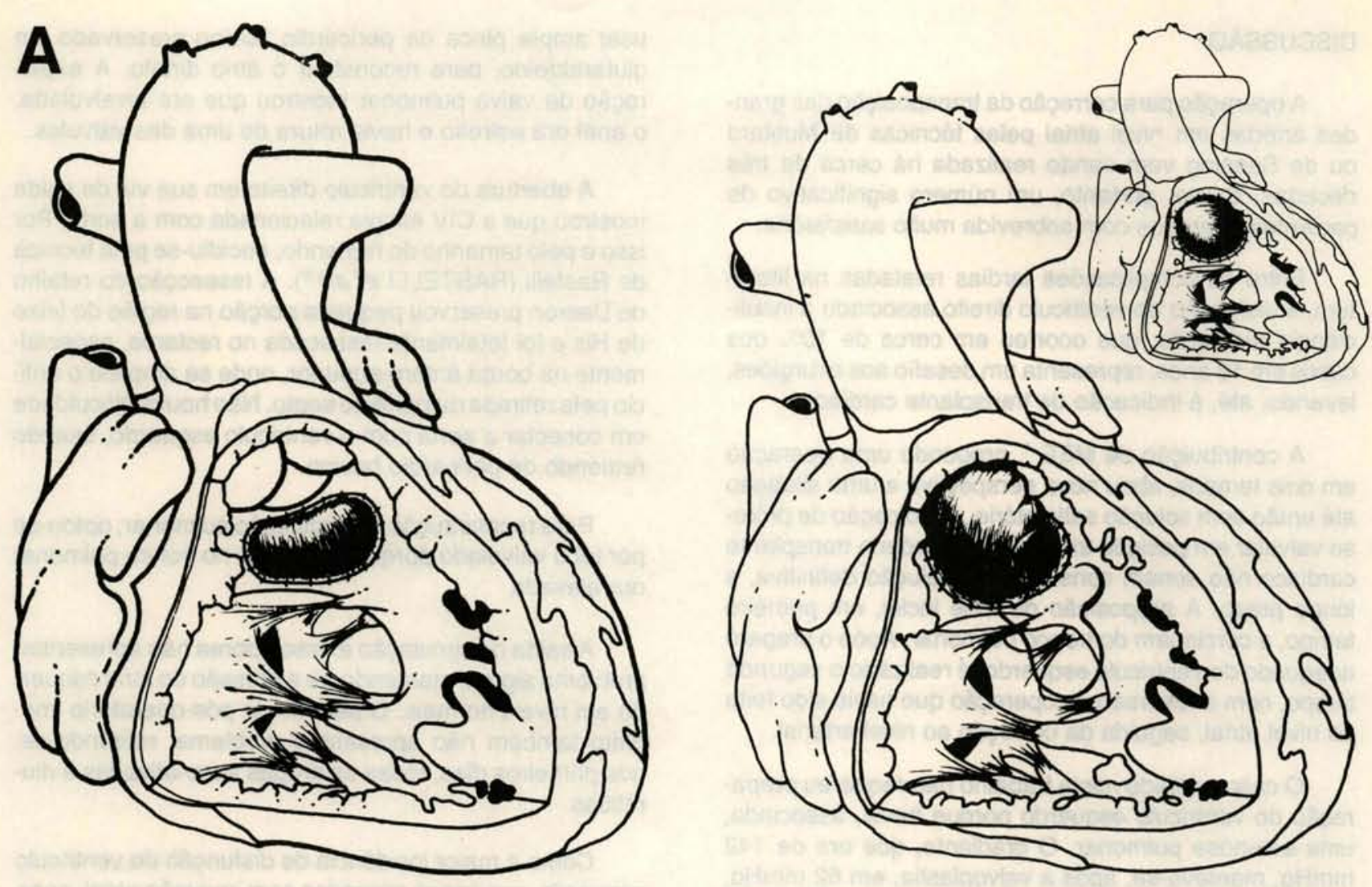

Fig. 2-Redirecionamento do fluxo de VE para aorta. No detalhe, aspecto final do remendo.

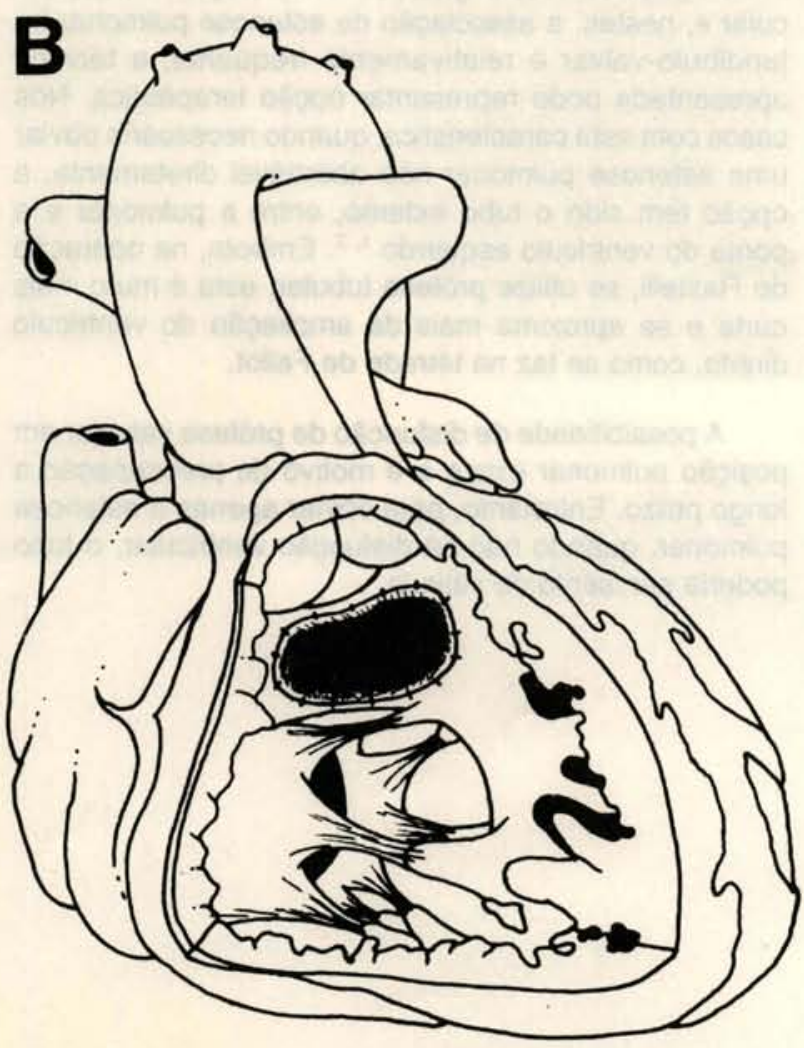

Fig. 1 - A: aspecto da CIV ocluida com aorta no ventriculo direito. B: remendo que ocluia a CIV removido.

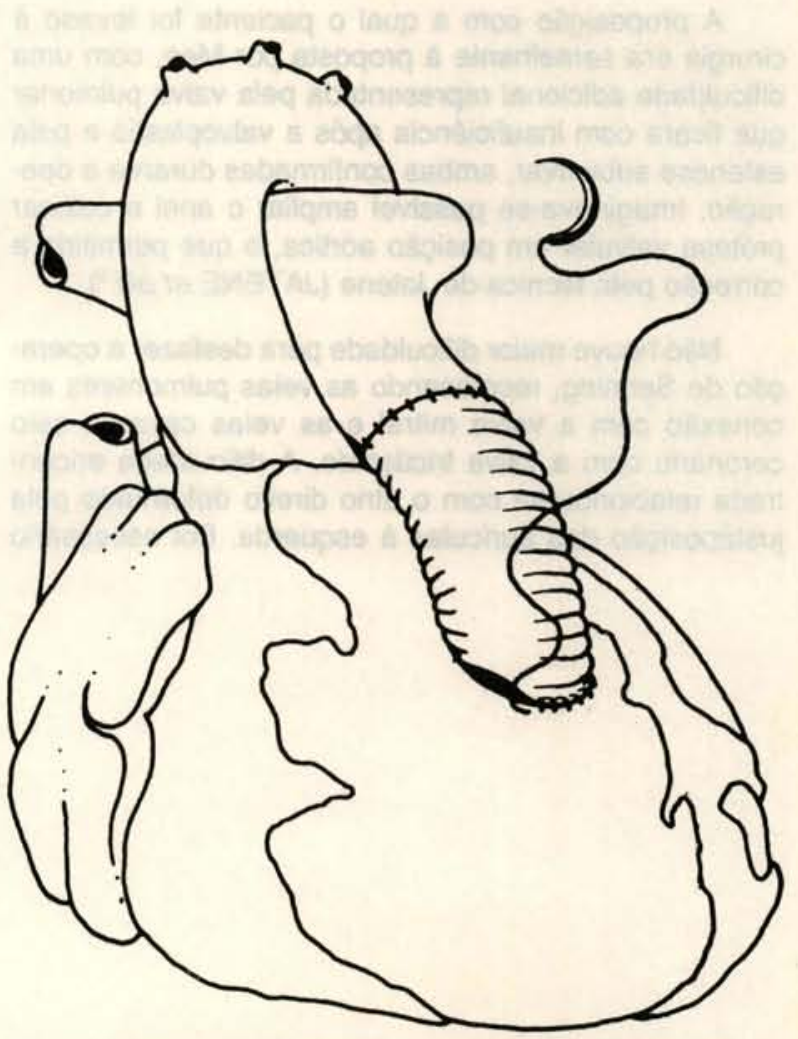

Fig. 3-Reconstrução da circulação pulmonar com tubo valvulado. 
JATENE, A. D.; JATENE, F. B.; JATENE, M. B.; IKARI, N.; MACHADO, D. M. A. - Correção cirúrgica para reversão de operaçāo de Senning em caso de falência ventricular direita. Rev. Bras. Cir. Cardiovasc., 6(1): 49-53, 1991.

\section{DISCUSSĀO}

A operação para correção da transposiçāo das grandes artérias em nível atrial pelas técnicas de Mustard ou de Senning vem sendo realizada há cerca de três décadas. Existe, portanto, um número significativo de pacientes operados com sobrevida muito satisfatória.

Entre as complicaçōes tardias relatadas na literatura, a disfunção do ventrículo direito associada a insuficiência tricúspide, que ocorreu em cerca de $10 \%$ dos casos em 10 anos, representa um desafio aos cirurgiōes, levando, até, à indicação de transplante cardíaco.

A contribuição de $\mathrm{MEE}^{4}$, propondo uma operação em dois tempos, abriu nova perspectiva a uma situação até entăo sem solução satisfatória. A indicação de prótese valvular em posição tricúspide e o próprio transplante cardíaco não seriam considerados solução definitiva, a longo prazo. A proposição de Mee inclui, em primeiro tempo, a cerclagem do tronco pulmonar. Após o preparo adequado do ventrículo esquerdo, é realizado o segundo tempo, com a reversāo da operação que havia sido feita ao nível atrial, seguida da correção ao nível arterial.

O caso relatado neste trabalho não requereu preparação do ventrículo esquerdo porque havia, associada, uma estenose pulmonar. $\mathrm{O}$ gradiente, que era de 142 $\mathrm{mmHg}$, manteve-se, após a valvoplastia, em $62 \mathrm{mmHg}$, suficiente para manter o condicionamento do ventrículo esquerdo.

A proposição com a qual o paciente foi levado à cirurgia era semelhante à proposta por Mee, com uma dificuldade adicional representada pela valva pulmonar que ficara com insuficiência após a valvoplastia e pela estenose subvalvar, ambas confirmadas durante a operação. Imaginava-se possível ampliar o anel e colocar prótese valvular em posição aórtica, o que permitiria a correção pela técnica de Jatene (JATENE et alii ${ }^{3}$ ).

Não houve maior dificuldade para desfazer a operaçăo de Senning, recolocando as veias pulmonares em conexão com a valva mitral e as veias cavas e seio coronário com a valva tricúspide. A dificuldade encontrada relacionou-se com o átrio direito deformado pela justaposição das aurículas à esquerda. Foi necessário usar ampla placa de pericárdio bovino preservado em glutaraldeído, para reconstruir o átrio direito. A exploração da valva pulmonar mostrou que era bivalvulada, o anel era estreito e havia rotura de uma das válvulas.

A abertura do ventrículo direito em sua via de saída mostrou que a CIV estava relacionada com a aorta. Por isso e pelo tamanho do remendo, decidiu-se pela técnica de Rastelli (RASTELLI et alii ${ }^{6}$ ). A ressecção do retalho de Dacron preservou pequena porção na região do feixe de His e foi totalmente ressecada no restante, especialmente na borda ântero-superior, onde se ampliou o oriffcio pela retirada de parte do septo. Não houve dificuldade em conectar a aorta com o ventrículo esquerdo, usando remendo de pericárdio bovino.

Para reconstrução da circulação pulmonar, optou-se por tubo valvulado porque a pressão no tronco pulmonar era elevada.

A saída da circulação extracorpórea não apresentou problema algum, mantendo-se a pressão do átrio esquerdo em níveis normais. O período de pós-operatório imediato também não apresentou problema, retirando-se, nos primeiros dias, todas as drogas vaso-ativadas e diuréticas.

Como a maior incidência de disfunção do ventrículo esquerdo, nos casos operados com inversão atrial, ocorre em pacientes com grande comunicação interventricular e, nestes, a associação de estenose pulmonar infundíbulo-valvar é relativamente freqüente, a técnica apresentada pode representar opção terapêutica. Nos casos com esta característica, quando necessário obviar uma estenose pulmonar não abordável diretamente, a opção tem sido o tubo externo, entre a pulmonar e a ponta do ventrículo esquerdo ${ }^{1,2}$. Embora, na operação de Rastelli, se utilize prótese tubular, esta é muto mais curta e se aproxima mais da ampliação do ventrículo direito, como se faz na tétrade de Fallot.

A possibilidade de disfunção de prótese valvular em posição pulmonar existe e é motivo de preocupação a longo prazo. Entretanto, para obviar apenas a estenose pulmonar, quando não há disfunção ventricular, o tubo poderia ser isento de válvula. 
JATENE, A. D.; JATENE, F. B.; JATENE, M. B.; IKARI, N.; MACHADO, D. M. A. - Correção cirúrgica para reversão de operação de Senning em caso de falência ventricular direita. Rev. Bras. Cir. Cardiovasc., 6(1): 49-53, 1991.

\section{RBCCV 44205-132}

JATENE, A. D.; JATENE, F. B.; JATENE, M. B.; IKARI, N.; MACHADO, D. M. A. - Surgical correction for atrial inversion in Senning operation in case of right ventricular dysfunction. Rev. Bras. Cir. Cardiovasc., $6(1)$ : 49-53, 1991.

ABSTRACT: A new proposal for the treatment of right ventricular dysfunction in late postoperative period of patients submitted to Senning operation is presented. The technique could be used in cases originally with transposition of the great arteries associated with pulmonary stenosis and a large VSD. A 12-year-old patient, who had been submitted, 8 years before, to an atrial inversion associated with closure of a large VSD and relieve of a pulmonary stonosis was submitted to this new operation. Senning operation was undone thus reestablishing atrio-ventricular concordance; the patch used for closure of VSD was removed, and the aorta was connected to the left-ventricle. Pulmonary artery was cut transversally, adjacent to the valvar horizontal plane. The proximal extremity was sutured and the distal was connected to the right ventricle through a valvar tube graft of bovine pericardium. The clinical course was uneventful and the patient improved from functional class IV to I/II.

DESCRIPTORS: Senning operation, reversion; Senning operation, late complications; ventricular dysfunction, surgery.

\section{REFERÊNCIAS BIBLIOGRÁFICAS}

1 BATTISTESSA, S. \& DEVERALL, P. B. - Surgical relief of subpulmonary stenosis after previous Senning (or Mustard) operation for complete transposition. Int. J. Cardiol., 24: 230-233, 1989.

2 CRUPI, G.; PILLAI, R.; PARENZAN, L.; LINCOLN, C. Surgical treatment of subpulmonary obstruction in transposition of the great arteries by means of a left ventricle pulmonary artery conduit. J. Thorac. Cardiovasc. Surg., 89: 907-913, 1985.

3 JATENE, A. D.; FONTES, V. F.; PAULISTA, P. P.; SOUZA, L. C. B.; NEGER, F.; GALANTIER, M.; SOUSA, J. E. M. R. - Anatomic correction of transposition of the great vessels. J. Thorac. Cardiovasc. Surg., 73: 363-370, 1976.

4 MEE, R. B. B. - Severe right ventricular failure after Mustard or Senning operation. Two-stage repair: pulmonary artery banding and switch. J. Thorac. Cardiovasc. Surg., 92: 385-390, 1986.

5 MUSTARD, W. T. - Successful two-stage correction of transposition of the great vessels. Surgery, 55: 469-472, 1964.

6 RASTELLI, G. C.; WALLACE, R. B.; ONGLWY, P. A. Complete repair of transposition of the great arteries with pulmonary stenosis: a review and report of a case corrected by using a new surgical technique. Circulation, 39: 83-95, 1969.

7 SAXEMA, A.; FONG, L. V.; OLGILVE, B. C.; KEETON, B. R. - Use of balloon dilatation to tear supravalvar stenosis developing after anatomical correction for complete transposition. Br. Heart J., 64: 151-155, 1990.

8 SENNING, A. - Surgical correction of transposition of the great vessels. Surgery, 45: 966-980, 1959.

\section{Discussão}

DR. IVO A. NESRALLA
Porto Alegre, RS

Os pacientes portadores de transposição das grandes artérias apresentam, na sua história natural, graus variáveis de disfunção ventricular direita, o que se traduz por volume diastólico elevado. Após a correção em nível atrial, 5 a $10 \%$ desses pacientes apresentam insuficiência ventricular direita grave, cujas possíveis causas sāo: 1) 0 arranjo anatômico das fibras musculares do ventrículo direito é diferente daquele do ventrículo esquerdo; 2) a vascularização do ventrículo direito é menor do que a do ventrículo esquerdo. Até o surgimento da operação de Jatene, crianças como essas, com importante insuficiência do ventrículo direito, eram candidatas a transplante cardíaco, ou cardiopulmonar. Com o advento da sua operação, Prof. Adib, e a forma técnica engenhosa e original como foi conduzido o caso aqui apresentado, um novo horizonte se abre para este tipo de complicação da correção em nível atrial.

\section{PROF. JATENE \\ (Encerrando)}

Obrigado ao Dr. Nesralla. Realmente esta perspectiva de reversão das técnicas de inversão atrial já vêm sendo realizadas. No caso presente, a operaçăo de Jatene não poderia ser considerada, porque havia alteração do anel e da valva pulmonar. Por isso, a sua reversão para a técnica de Rastelli, até aqui não descrita, nos pareceu proposição mais adequada que o transplante cardíaco. 